\section{OTR.04 - Traces of immunoediting during myeloproliferative neoplasm to secondary acute myeloid leukemia transition}

Marco Antônio Pretti ${ }^{1}$; Luciana Carvalho Barros ${ }^{1 \star}$; Jackline Ayres ${ }^{2}$; Nicole Scherer ${ }^{1}$; Adelmo Daumas ${ }^{3}$; Cristiana Solza ${ }^{4}$; Esteban Braggio ${ }^{5}$; Ilana Zalcberg Renault ${ }^{1}$; Mariana Boroni ${ }^{1}$; Martín Hernán Bonamino ${ }^{6}$.

\section{INCa;}

2Fiocruz - Fundação Oswaldo Cruz;

3Hospital Universitário Antônio Pedro;

4Hospital Universitário Pedro Ernesto;

5Mayo Clinic;

6INCa / Fiocruz.

\section{Introduction:}

Immunological response against transformed cells are antigen specific and capable of controlling tumor development. However, in some cases, tumors escape immunologic surveillance by losing the more immunogenic variants. This impacts the neoantigen (NeoAg) burden, the amount of new antigens generated by coding change somatic mutations. Myeloproliferative neoplasm (MPN) are diseases impacting the myeloid compartment in which mutations in driver genes and/or epigenetic modulators lead to expansion of mature myeloid derived populations. Such diseases can eventually progress to secondary acute myeloid leukemia (sAML), having a dismal prognosis. Limited data has been generated regarding their neoantigen profile and how these profiles are modulated during the MPN $\rightarrow$ sAML transition.

\section{Objective:}

Search for immunoediting evidence in tumor progression analyzing the whole exome of samples collected before and after the leukemic transformation process to generate in silico predictions on neoantigen burden.

\section{Methodology:}

Whole exome of paired samples from three patients diagnosed with Essential Thrombocythemia that evolved to sAML were analyzed following GATK best practices. Variant calling was performed by Varscan2.4.3 using reference genome GRCh37. Variants were annotated with Annovar and peptide affinity to HLA-I was predicted by netMHCpan. HLA-I allele identification was performed by Optitype. Expression data was obtained from TARGET-AML and CCLE (MPN).

\section{Results:}

We found a decrease in mutation burden (Unique Patient Number - UPN719: 162 to 109 ; UPN883: 167 to 123 ; UPN249: 182 to 110) and consequently in NeoAg burden (13 to $12 ; 21$ to $16 ; 18$ to 15 ) during the NMP $\rightarrow$ sAML transition. Unlike the other patients, UPN249 possess two NeoAg exclusive to sAML phase derived from a TP53 mutation, which may benefit tumor growth despite its immunogenicity. Binding affinity predictions didn't indicate any preferential loss of NeoAg with higher affinity. Clonality of NeoAg generating variants evidenced changes in clonal populations with reduction in variant allele frequency (VAF) of NeoAg with high HLA-I affinity (UPN719 and UPN249). The increase in VAF was preferentially observed in NeoAg with low affinity (UPN719 and UPN249) and/or driver genes (UPN719: JAK2; UPN249: CALR and TP53). As an additional potential immune escape mechanism, we found a NeoAg in one deleted region after transition in UPN249. UPN883 and UPN249 carry a frameshift mutation in CALR affecting NeoAg presentation. Additionally, other mutations in antigen processing and presenting pathways were detected in UPN719 and UPN883. Analysis of expression levels on databases indicates that most of the neoantigens identified are located in genes with low expression in AML samples.

\section{Conclusion:}

We found a reduction in NeoAg burden during MPN $\rightarrow$ sAML transitions as well as changes in VAF of NeoAg. These reductions advocate in favor of immune mediated impact on NeoAg repertoire, indicating potential ongoing immune editing process. Moreover, deficiency in antigen presentation proteins and chromosomal losses impacting NeoAg expression may impact the immunogenicity landscape of MPN $\rightarrow$ sAML transitions.

Keywords: Immunoediting; Myeloproliferative Neoplasms; Neoantigen 